\title{
Arbitrarily high-order energy-preserving methods for simulating the gyrocenter dynamics of charged particles
}

\author{
Luigi Brugnano $^{\text {a }} \quad$ Felice Iavernaro $^{b} \quad$ Ruili Zhang $^{\mathrm{c}}$ \\ a) Dipartimento di Matematica e Informatica "U. Dini", Università di Firenze, 50134 Firenze, Italy. \\ luigi.brugnano@unifi.it \\ b) Dipartimento di Matematica, Università di Bari, 70125 Bari, Italy. \\ felice.iavernaro@uniba.it \\ c) School of Science, Beijing Jiaotong University, Beijing, 100044, China.
}

rlzhang@ustc.edu.cn

\begin{abstract}
Gyrocenter dynamics of charged particles plays a fundamental role in plasma physics. In particular, accuracy and conservation of energy are important features for correctly performing long-time simulations. For this purpose, we here propose arbitrarily high-order energy conserving methods for its simulation. The analysis and the efficient implementation of the methods are fully described, and some numerical tests are reported.
\end{abstract}

Keywords: gyrocenter dynamics, energy-conserving methods, Poisson problems, line integral methods, Hamiltonian Boundary Value Methods, HBVMs.

MSC: 65P10, 65L05.

\section{Introduction}

The motion of an electrically charged particle, such as an electron or ion, in a plasma plunged in an electro-magnetic field can be treated as the superposition of a relatively fast circular motion (gyromotion) around a point called the gyrocenter, and a relatively slow drift of this point. Its numerical simulation is an important issue in plasma physics but brings a number of difficulties. Not only does the problem exhibit different time-scales due to the fast gyromotion and the slow gyrocenter motion, but it also is multi-scale in space, since the fast gyromotion is small-scale, whereas the slow gyrocenter motion is large-scale. However, when we pay attention to the orbit of a charged particle, the fast gyromotion usually has not much information and, moreover, it is time consuming in numerical simulations. Consequently, we shall focus on the orbits of the gyrocenter, thus only considering the gyrocenter dynamics of a charged particle.

The mathematical description of the gyrocenter dynamics of a charged particle has been at first given by R.G. Littlejohn [27]. In more details, we shall consider the case of time-independent magnetic and electric fields, respectively given by

$$
B(x)=\nabla \times A(x) \quad \text { and } \quad E(x)=-\nabla \phi(x), \quad x \in \mathbb{R}^{3},
$$


with $\phi(x)$ the electric potential and

$$
A(x) \equiv\left(\begin{array}{c}
A_{1}(x) \\
A_{2}(x) \\
A_{3}(x)
\end{array}\right)
$$

the magnetic vector potential. In such a case, by setting $x=\left(x_{1}, x_{2}, x_{3}\right)^{\top} \in \mathbb{R}^{3}$ the position of the gyrocenter, defining ${ }^{1}$

$$
b(x) \equiv\left(\begin{array}{c}
b_{1}(x) \\
b_{2}(x) \\
b_{3}(x)
\end{array}\right):=\frac{B(x)}{\|B(x)\|}
$$

as the unit vector in the direction of the magnetic field, along which the gyrocenter moves with velocity $u$, and setting

$$
y:=\left(\begin{array}{l}
x \\
u
\end{array}\right) \in \mathbb{R}^{4}
$$

the equations describing the gyrocenter dynamics of the charged particle can be written as

$$
\dot{y}=K(y)^{-1} \nabla H(y),
$$

where:

- the Hamiltonian function $H(y)$ is given by

$$
H(y)=\frac{1}{2} u^{2}+\mu\|B(x)\|+\phi(x),
$$

with $\mu$ a given constant representing an adiabatic invariant;

- $K^{-1}(y)$ is the following skew-symmetric matrix,

$$
K^{-1}(y)=\frac{1}{\left|b(x)^{\top} a(y)\right|}\left(\begin{array}{cccc}
0 & -b_{3}(x) & b_{2}(x) & a_{1}(y) \\
b_{3}(x) & 0 & -b_{1}(x) & a_{2}(y) \\
-b_{2}(x) & b_{1}(x) & 0 & a_{3}(y) \\
-a_{1}(y) & -a_{2}(y) & -a_{3}(y) & 0
\end{array}\right),
$$

with $b(x)$ the vector defined in $(3)$ and

$$
a(y) \equiv\left(\begin{array}{l}
a_{1}(y) \\
a_{2}(y) \\
a_{3}(y)
\end{array}\right):=\nabla \times[A(x)+u b(x)]=B(x)+u \nabla \times b(x) .
$$
form

Consequently, without loss of generality, the problem we want to solve can be assumed in the

$$
\dot{y}=S(y) \nabla H(y)=: f(y), \quad t \in[0, h], \quad y(0)=y_{0}, \quad S(y)=-S(y)^{\top},
$$

where $h$ is the timestep used and, for sake of brevity, the notation $S(y)=K^{-1}(y)$ has been used. Clearly, for such a problem, the energy $H$ is conserved along the solution, since

$$
\dot{H}(y)=\nabla H(y)^{\top} \dot{y}=\nabla H(y)^{\top} S(y) \nabla H(y)=0,
$$

\footnotetext{
${ }^{1}$ Hereafter, $\|\cdot\|$ will denote the Euclidean norm.
} 
due to the skew-symmetry of $S(y)$. Energy-conserving methods for problems in this form have been already considered, e.g., in [22, 5, 28. Here, the derivation and analysis of the methods will be done within the framework of line integral methods, namely methods defined by a suitable smooth path $\sigma$ such that:

$$
\sigma(0)=y_{0}, \quad y_{1}:=\sigma(h),
$$

with $y_{1}$ the approximation to $y(h)$, and ${ }^{2}$

$$
H\left(y_{1}\right)-H\left(y_{0}\right)=H(\sigma(h))-H(\sigma(0))=h \int_{0}^{1} \nabla H(\sigma(c h))^{\top} \dot{\sigma}(c h) \mathrm{d} c=0 .
$$

In such a case, the conservation of $H$ will no more require the integrand to be identically zero, as is the case for $y(t)$ (see (10), thus leaving much more freedom in the choice of the path $\sigma$. Line integral methods have been at first introduced for deriving energy-conserving methods for Hamiltonian problems [24, 25, 26, 11, 12, 13, 15, resulting in the class of energy-conserving methods named Hamiltonian Boundary Value Methods (HBVMs), and have then been developed along several directions (see, e.g., the monograph [8] or the review papers [9, 7]), including their use as spectral methods in time [20, 10, 1, 2].

With these premises, in Section 2 we derive and analyse, at a continuous level, polynomial approximations able to exactly conserve the energy $H$; fully discrete methods are then derived and studied in Section 3 , where we also sketch the efficient solution of the generated discrete problems; finally, a few numerical tests are reported in Section 4 , along with some concluding remarks.

\section{Derivation of the method}

The approach used to derive the method is based on a suitable modification of the arguments used in [15, 21. To begin with, let us expand the gradient of $H$ along the orthonormal Legendre polynomial basis on $[0,1]$ :

$$
P_{i} \in \Pi_{i}, \quad \int_{0}^{1} P_{i}(x) P_{j}(x) \mathrm{d} x=\delta_{i j} . \quad i, j=0,1, \ldots,
$$

where, as usual, $\Pi_{i}$ is the set of polynomials of degree at most $i$, and $\delta_{i j}$ is the Kronecker delta. Moreover, for sake of simplicity, we shall hereafter assume that both $\nabla H(y(t))$ and $S(y(t))$ admit a Taylor expansion at $t=0$. Consequently, one has

$$
\nabla H(y(c h))=\sum_{j \geq 0} P_{j}(c) \gamma_{j}(y), \quad \gamma_{j}(y)=\int_{0}^{1} P_{j}(\tau) \nabla H(y(\tau h)) \mathrm{d} \tau, \quad j=0,1, \ldots,
$$

and, therefore, the equation in 9 can be rewritten as

$$
\dot{y}(c h)=S(y(c h)) \sum_{j \geq 0} P_{j}(c) \gamma_{j}(y), \quad c \in[0,1] .
$$

\footnotetext{
${ }^{2}$ The denomination line integral methods stems from the fact that one requires the vanishing of the line integral along the path $\sigma$, as prescribed by 12 .
} 
Next, let us consider the expansions

$$
P_{j}(c) S(y(c h))=\sum_{i \geq 0} P_{i}(c) \rho_{i j}(y), \quad \rho_{i j}(y)=\int_{0}^{1} P_{i}(\tau) P_{j}(\tau) S(y(\tau h)) \mathrm{d} \tau \equiv \rho_{j i}(y), \quad i, j=0,1, \ldots
$$

Eventually, we obtain that the equation in $(9)$ can be rewritten, by virtue of $(14)-(16)$, as

$$
\dot{y}(c h)=\sum_{i, j \geq 0} P_{i}(c) \rho_{i j}(y) \gamma_{j}(y) \equiv \sum_{i \geq 0} P_{i}(c) \Gamma_{i}(y), \quad c \in[0,1], \quad \Gamma_{i}(y)=\sum_{j \geq 0} \rho_{i j}(y) \gamma_{j}(y),
$$

from which, integrating term by term, and imposing the initial condition $y(0)=y_{0}$, one has:

$$
y(c h)=y_{0}+h \sum_{i \geq 0} \int_{0}^{c} P_{i}(x) \mathrm{d} x \Gamma_{i}(y), \quad c \in[0,1] .
$$

Remark 1 It is clear, from (17), that $\Gamma_{i}(y)$ is nothing but the $i$-th Fourier coefficient in the expansion of the right-hand side of (9) along the Legendre basis (13), i.e.,

$$
f(y(c h))=\sum_{i \geq 0} P_{i}(c) \Gamma_{i}(y), \quad c \in[0,1] .
$$

We need the following preliminary results.

Lemma 1 Let $g:[0, h] \rightarrow V$, with $V$ a vector space, admit a Taylor expansion at 0 . Then,

$$
\int_{0}^{1} P_{j}(c) g(c h) \mathrm{d} c=O\left(h^{j}\right), \quad j=0,1, \ldots
$$

Proof See [15, Lemma 1].

Lemma $2 \gamma_{j}(y)=O\left(h^{j}\right), \rho_{i j}(y)=-\rho_{i j}(y)^{\top}=O\left(h^{|j-i|}\right)$.

Proof The first point follows from Lemma 1, the second from [21, Corollary 1] and the skewsymmetry of $S(y)$.

Lemma $3 \Gamma_{i}(y)=O\left(h^{i}\right)$.

Proof The statement follows from Lemma 1 by taking into account 19.

In order to obtain a polynomial approximation of degree $s$ to $y$, we truncate the series in (14) and (16) to finite sums, thus getting

$$
\dot{\sigma}(c h)=\sum_{i=0}^{s-1} P_{i}(c) \Gamma_{i}^{s}(\sigma), \quad c \in[0,1], \quad \Gamma_{i}^{s}(\sigma)=\sum_{j=0}^{s-1} \rho_{i j}(\sigma) \gamma_{j}(\sigma), \quad i=0, \ldots, s-1,
$$

with $\gamma_{j}(\sigma)$ and $\rho_{i j}(\sigma)$ formally still defined by (14) and (16), respectively, upon replacing $y$ by $\sigma$. It is straightforward to verify that Lemma 2 continues to hold for the Fourier coefficients $\rho_{i j}(\sigma)$ and $\gamma_{j}(\sigma)$. Moreover, similarly as for Lemma 3 . now one has the following result which holds true for $\Gamma_{i}^{s}(\sigma)$. 
Lemma 4 For all $i=0, \ldots, s-1$, one has: $\Gamma_{i}^{s}(\sigma)=O\left(h^{i}\right), \Gamma_{i}(\sigma)-\Gamma_{i}^{s}(\sigma)=O\left(h^{2 s-i}\right)$.

Proof One has, by virtue of Lemma 2, and for $i=0, \ldots, s-1$ :

$$
\Gamma_{i}^{s}(\sigma)=\sum_{j=0}^{s-1} \rho_{i j}(\sigma) \gamma_{j}(\sigma)=\sum_{j=0}^{i} \underbrace{O\left(h^{i-j}\right) O\left(h^{j}\right)}_{=O\left(h^{i}\right)}+\sum_{j=i+1}^{s-1} \underbrace{O\left(h^{j-i}\right) O\left(h^{j}\right)}_{=O\left(h^{2 j-i}\right)}=O\left(h^{i}\right) .
$$

Similarly, for $i<s$, one obtains:

$$
\Gamma_{i}(\sigma)-\Gamma_{i}^{s}(\sigma)=\sum_{j \geq s} \rho_{i j}(\sigma) \gamma_{j}(\sigma)=\sum_{j \geq s} \underbrace{O\left(h^{j-i}\right) O\left(h^{j}\right)}_{=O\left(h^{2 j-i}\right)}=O\left(h^{2 s-i}\right) .
$$

Integrating term by term $(20)$, and imposing the initial condition $\sigma(0)=y_{0}$, one then obtains the polynomial approximation of degree $s$

$$
\sigma(c h)=y_{0}+h \sum_{i=0}^{s-1} \int_{0}^{c} P_{i}(x) \mathrm{d} x \Gamma_{i}^{s}(\sigma), \quad c \in[0,1],
$$

with the approximation to $y(h)$ given, by considering that $\int_{0}^{1} P_{i}(x) \mathrm{d} x=\delta_{i 0}$ (see 13$)$, by:

$$
y_{1}:=\sigma(h)=y_{0}+h \Gamma_{0}^{s}(\sigma) .
$$

In so doing, the requirements (11) are fulfilled.

\subsection{Analysis}

Let us now study the properties of the approximation procedure 20122. To begin with, let us now prove that also the requirement $[12$ holds true, besides (11).

Theorem 1 For the polynomial approximation defined by (20)-22), one has: $H\left(y_{1}\right)=H\left(y_{0}\right)$, i.e., the method is energy-conserving.

Proof In fact, according to 12 one obtains:

$$
\begin{aligned}
& H\left(y_{1}\right)-H\left(y_{0}\right)=H(\sigma(h))-H(\sigma(0))=h \int_{0}^{1} \nabla H(\sigma(c h))^{\top} \dot{\sigma}(c h) \mathrm{d} c \\
& =h \int_{0}^{1} \nabla H(\sigma(c h))^{\top} \sum_{i=0}^{s-1} P_{i}(c) \Gamma_{i}^{s}(\sigma) \mathrm{d} c=h \sum_{i=0}^{s-1} \int_{0}^{1} \nabla H(\sigma(c h))^{\top} P_{i}(c) \mathrm{d} c \Gamma_{i}^{s}(\sigma) \\
& =h \sum_{i=0}^{s-1} \gamma_{i}(\sigma)^{\top} \Gamma_{i}^{s}(\sigma)=h \sum_{i, j=0}^{s-1} \gamma_{i}(\sigma)^{\top} \rho_{i j}(\sigma) \gamma_{j}(\sigma)=0,
\end{aligned}
$$

due to the skew-symmetry of $\rho_{i j}(\sigma)$.

Next, let us discuss the accuracy of the approximation $(22)$ to $y(h)$, adapting the arguments of [15. Theorem 1]. Preliminarily, let us denote by $y\left(t, t^{*}, y^{*}\right)$ the solution of the problem (see (9))

$$
\dot{y}=f(y), \quad t \in\left[t^{*}, h\right], \quad y\left(t^{*}\right)=y^{*},
$$


also recalling the following well-known perturbation results,

$$
\frac{\partial}{\partial y^{*}} y\left(t, t^{*}, y^{*}\right)=\Phi\left(t, t^{*}\right), \quad \frac{\partial}{\partial t^{*}} y\left(t, t^{*}, y^{*}\right)=-\Phi\left(t, t^{*}\right) f\left(y^{*}\right),
$$

where $\Phi\left(t, t^{*}\right)$ is the fundamental matrix solution of the variational problem associated to 23 .

Theorem 2 For the polynomial approximation defined by (20)-(22), one has: $y_{1}-y(h)=O\left(h^{2 s+1}\right)$, where $y(t)$ is the solution of problem (9) $:^{3}$

Proof By virtue of Lemmas 1, 3, and 4 , from 23)-24, and considering that, according to 19$]$,

$$
f(\sigma(c h))=\sum_{j \geq 0} P_{j}(c) \Gamma_{i}(\sigma), \quad c \in[0,1]
$$

one has:

$$
\begin{aligned}
y_{1} & -y(h)=y\left(h, h, y_{1}\right)-y\left(h, 0, y_{0}\right)=y(h, h, \sigma(h))-y(h, 0, \sigma(0))=\int_{0}^{h} \frac{\mathrm{d}}{\mathrm{d} t} y(h, t, \sigma(t)) \mathrm{d} t \\
= & \int_{0}^{h}\left[\left.\frac{\partial}{\partial t^{*}} y\left(h, t^{*}, \sigma(t)\right)\right|_{t^{*}=t}+\left.\frac{\partial}{\partial y^{*}} y\left(h, t, y^{*}\right)\right|_{y^{*}=\sigma(t)} \dot{\sigma}(t)\right] \mathrm{d} t \\
= & \int_{0}^{h}[-\Phi(h, t) f(\sigma(t))+\Phi(h, t) \dot{\sigma}(t)] \mathrm{d} t=h \int_{0}^{1} \Phi(h, c h)[\dot{\sigma}(c h)-f(\sigma(c h))] \mathrm{d} c \\
= & h \sum_{i=0}^{s-1} \underbrace{\int_{0}^{1} P_{i}(c) \Phi(h, c h) \mathrm{d} c}_{=O\left(h^{i}\right)} \underbrace{\left[\Gamma_{i}^{s}(\sigma)-\Gamma_{i}(\sigma)\right]}_{=O\left(h^{2 s-i}\right)}-h \sum_{i \geq s}^{\int_{0}^{\int_{0}^{1} P_{i}(c) \Phi(h, c h)} \mathrm{d} c \underbrace{\Gamma_{i}(\sigma)}_{=O\left(h^{i}\right)}=O\left(h^{2 s+1}\right) .} .
\end{aligned}
$$

\section{Discretization}

As is clear, the polynomial approximation $20-22$ does not yet provide a numerical method. As matter of fact, quoting [23, p. 521], "as is well known, even many relatively simple integrals cannot be expressed in finite terms of elementary functions, and thus must be evaluated by numerical methods". In the present setting, this means that we need to approximate the Fourier coefficients

$$
\rho_{i j}(\sigma)=\int_{0}^{1} P_{i}(c) P_{j}(c) S(\sigma(c h)) \mathrm{d} c, \quad \gamma_{j}(\sigma)=\int_{0}^{1} P_{j}(c) \nabla H(\sigma(c h)) \mathrm{d} c, \quad i, j=0, \ldots, s-1,
$$

by using suitable quadratures. For this purpose, we shall use:

- a Gauss-Legendre formula of order $2 k_{1}$, with $k_{1} \geq s$, to approximate the former coefficients, whose abscissae and weights shall be denoted by

$$
\hat{c}_{\ell}, \hat{b}_{\ell}, \quad \ell=1, \ldots, k_{1},
$$

respectively;

\footnotetext{
${ }^{3}$ I.e., the method has order $2 s$.
} 
- a Gauss-Legendre formula of order $2 k_{2}$, with $k_{2} \geq s$, to approximate the latter coefficients, whose abscissae and weights shall be denoted by

$$
c_{\ell}, b_{\ell}, \quad \ell=1, \ldots, k_{2},
$$

respectively.

We recall that, due to the symmetry of the abscissae $(26)$ and $(27)$, one has:

$$
\hat{c}_{\ell}=1-\hat{c}_{k_{1}-\ell+1}, \quad \hat{b}_{\ell}=\hat{b}_{k_{1}-\ell+1}, \quad \ell=1, \ldots, k_{1},
$$

and

$$
c_{\ell}=1-c_{k_{2}-\ell+1}, \quad b_{\ell}=b_{k_{2}-\ell+1}, \quad \ell=1, \ldots, k_{2} .
$$

As a result, in place of the polynomial $\sigma$ defined at 20]-21), we shall have a, generally different, polynomial $u \in \Pi_{s}$, such that:

$$
\dot{u}(c h)=\sum_{i=0}^{s-1} P_{i}(c) \hat{\Gamma}_{i}, \quad c \in[0,1], \quad \hat{\Gamma}_{i}=\sum_{j=0}^{s-1} \hat{\rho}_{i j} \hat{\gamma}_{j}, \quad i=0, \ldots, s-1,
$$

with the approximate Fourier coefficients

$$
\hat{\rho}_{i j}=\sum_{\ell=1}^{k_{1}} \hat{b}_{\ell} P_{i}\left(\hat{c}_{\ell}\right) P_{j}\left(\hat{c}_{\ell}\right) S\left(u\left(c_{\ell} h\right)\right) \equiv \hat{\rho}_{j i}, \quad \hat{\gamma}_{j}=\sum_{\ell=1}^{k_{2}} b_{\ell} P_{j}\left(c_{\ell}\right) \nabla H\left(u\left(c_{\ell} h\right)\right), \quad i, j=0, \ldots, s-1,
$$

in place of (25). Consequently, one obtains

$$
u(c h)=y_{0}+h \sum_{i=0}^{s-1} \int_{0}^{c} P_{i}(x) \mathrm{d} x \hat{\Gamma}_{i}, \quad c \in[0,1]
$$

with the new approximation given by (see 22 )

$$
y_{1}:=u(h) \equiv y_{0}+h \hat{\Gamma}_{0} .
$$

For later use, let us recall that the errors in the quadratures $(31)$ are given by (see $(25))^{4}$

$$
\hat{\rho}_{i j}-\rho_{i j}(u)=\hat{\Delta}_{i j}(h) \equiv\left\{\begin{array}{cl}
0, & \text { if } S \in \Pi_{\nu} \text { with } \nu<\left[2\left(k_{1}-s\right)+1\right] / s, \\
O\left(h^{2 k_{1}-i-j}\right), & \text { otherwise. }
\end{array}\right.
$$

and

$$
\hat{\gamma}_{j}-\gamma_{j}(u)=\Delta_{j}(h) \equiv\left\{\begin{array}{cl}
0, & \text { if } H \in \Pi_{\nu} \text { with } \nu \leq 2 k_{2} / s, \\
O\left(h^{2 k_{2}-j}\right), & \text { otherwise. }
\end{array}\right.
$$

Remark 2 As is clear from (34) and (35), when both the entries of the matrix $S$ and the energy $H$ in (9) are polynomials, we can exactly compute the Fourier coefficients, by choosing $k_{1}$ and $k_{2}$ large enough, so that $u \equiv \sigma$. This, in turn, will be not a big computational issue since, as we shall see later, the discrete problem will always have dimension $s$, independently of $k_{1}$ and $k_{2}$.

\footnotetext{
${ }^{4}$ Hereafter, $S \in \Pi_{\nu}$ means that the entries of matrix $S$ in 9 are polynomials of degree at most $\nu$ in the argument.
} 
It is quite straightforward to prove the following properties, representing the discrete counterpart of Lemma 2 ,

$$
\forall k_{1}, k_{2} \geq s: \quad \hat{\gamma}_{j}=O\left(h^{j}\right), \quad \hat{\rho}_{i j}=-\hat{\rho}_{i j}^{\top}=O\left(h^{|j-i|}\right), \quad i, j=0, \ldots, s-1 .
$$

Moreover, the following result holds also true ${ }^{5}$

Lemma $5 \forall k_{1}, k_{2} \geq s: \quad \hat{\Gamma}_{i}=O\left(h^{i}\right), \quad \hat{\Gamma}_{i}-\Gamma_{i}(u)=O\left(h^{2 s-i}\right), \quad i=0, \ldots, s-1$.

Proof The first part of the statement follows from the second one and from Lemma 3 by considering that $i<s$. Next, by virtue of Lemma 4, one has:

$$
\hat{\Gamma}_{i}-\Gamma_{i}(u)=\hat{\Gamma}_{i}-\Gamma_{i}^{s}(u)+\Gamma_{i}^{s}(u)-\Gamma_{i}(u)=\hat{\Gamma}_{i}-\Gamma_{i}^{s}(u)+O\left(h^{2 s-i}\right) .
$$

Moreover, from (20) and (34)-(35), one has:

$$
\begin{aligned}
\hat{\Gamma}_{i}-\Gamma_{i}^{s}(u) & =\sum_{j=0}^{s-1} \hat{\rho}_{i j} \hat{\gamma}_{j}-\rho_{i j}(u) \gamma_{j}(u)=\sum_{j=0}^{s-1}\left(\rho_{i j}(u)+\hat{\Delta}_{i j}(h)\right)\left(\gamma_{j}(u)+\Delta_{j}(u)\right)-\rho_{i j}(u) \gamma_{j}(u) \\
& =\sum_{j=0}^{s-1} \rho_{i j}(u) \Delta_{j}(u)+\hat{\Delta}_{i j}(h) \gamma_{j}(u)+\hat{\Delta}_{i j}(h) \Delta_{j}(h) .
\end{aligned}
$$

Considering that:

- $\rho_{i j}(u) \Delta_{j}(u)=O\left(h^{|j-i|+2 k_{2}-j}\right)$ and $|j-i|+2 k_{2}-j \geq 2 k_{2}-i \geq 2 s-i$;

- $\hat{\Delta}_{i j}(h) \gamma_{j}(u)=O\left(h^{2 k_{1}-i-j+j}\right)$ and $2 k_{1}-i \geq 2 s-i$;

- $\hat{\Delta}_{i j}(h) \Delta_{j}(h)=O\left(h^{2 k_{1}-i-j+2 k_{2}-j}\right)$ and $2\left(k_{1}+k_{2}-j\right)-i \geq 2 s-i$;

the statement then follows.

Definition 1 We call discrete line integral method with parameters $k_{1}, k_{2}, s$, in short $\operatorname{LIM}\left(k_{1}, k_{2}, s\right)$, the numerical method defined by (26)-(33).

\subsection{Analysis}

Let us now study the properties of the $\operatorname{LIM}\left(k_{1}, k_{2}, s\right)$ method 267-33). We start discussing the conservation of energy.

Theorem 3 For the $\operatorname{LIM}\left(k_{1}, k_{2}, s\right)$ method, with $k_{1}, k_{2} \geq s$, one has:

$$
H\left(y_{1}\right)-H\left(y_{0}\right)=\left\{\begin{array}{cl}
0, & \text { if } H \in \Pi_{\nu} \text { with } \nu \leq 2 k_{2} / s, \\
O\left(h^{2 k_{2}+1}\right), & \text { otherwise. }
\end{array}\right.
$$

\footnotetext{
${ }^{5}$ It represents the discrete counterpart of Lemma 4
} 
Proof In fact, by taking into account 14 and $30-36$, one obtains:

$$
\begin{aligned}
& H\left(y_{1}\right)-H\left(y_{0}\right)=H(u(h))-H(u(0))=h \int_{0}^{1} \nabla H(u(c h))^{\top} \dot{u}(c h) \mathrm{d} c \\
& =h \int_{0}^{1} \nabla H(u(c h))^{\top} \sum_{i=0}^{s-1} P_{i}(c) \hat{\Gamma}_{i} \mathrm{~d} c=h \sum_{i=0}^{s-1} \int_{0}^{1} \nabla H(u(c h))^{\top} P_{i}(c) \mathrm{d} c \hat{\Gamma}_{i} \\
& =h \sum_{i=0}^{s-1} \gamma_{i}(u)^{\top} \hat{\Gamma}_{i}=h \sum_{i, j=0}^{s-1} \gamma_{i}(u)^{\top} \hat{\rho}_{i j} \hat{\gamma}_{j}=h \sum_{i, j=0}^{s-1} \gamma_{i}(u)^{\top} \hat{\rho}_{i j}\left(\gamma_{j}(u)+\Delta_{j}(h)\right) \\
& =h \sum_{i, j=0}^{s-1} \gamma_{i}(u)^{\top} \hat{\rho}_{i j} \Delta_{j}(h) .
\end{aligned}
$$

When $H \in \Pi_{\nu}$, with $\nu \leq 2 k_{2} / s$, the quadrature error $\Delta_{j}(h)=0$. Conversely, one has:

$$
h \sum_{i, j=0}^{s-1} \gamma_{i}(u)^{\top} \hat{\rho}_{i j} \Delta_{j}(h)=h \sum_{i, j=0}^{s-1} \underbrace{\gamma_{i}(u)^{\top}}_{=O\left(h^{i}\right)} \overbrace{\hat{\rho}_{i j}}^{=O\left(h^{|j-i|}\right)} \underbrace{\Delta_{j}(h)}_{=O\left(h^{2 k_{2}-j}\right)}=O\left(h^{2 k_{2}+1}\right) .
$$

Concerning the order of accuracy, the following result holds true.

Theorem 4 For the $\operatorname{LIM}\left(k_{1}, k_{2}, s\right)$ method, if $k_{1}, k_{2} \geq s$ one has: $y_{1}-y(h)=O\left(h^{2 s+1}\right)$, where $y(t)$ is the solution of problem (9).

Proof Following similar steps as those used in the proof of Theorem 2, and taking into account (30) 36 and Lemma 5 , one has:

$$
\begin{aligned}
y_{1} & -y(h)=y\left(h, h, y_{1}\right)-y\left(h, 0, y_{0}\right)=y(h, h, u(h))-y(h, 0, u(0))=\int_{0}^{h} \frac{\mathrm{d}}{\mathrm{d} t} y(h, t, u(t)) \mathrm{d} t \\
= & \int_{0}^{h}\left[\left.\frac{\partial}{\partial t^{*}} y\left(h, t^{*}, u(t)\right)\right|_{t^{*}=t}+\left.\frac{\partial}{\partial y^{*}} y\left(h, t, y^{*}\right)\right|_{y^{*}=u(t)} \dot{u}(t)\right] \mathrm{d} t \\
= & \int_{0}^{h}[-\Phi(h, t) f(u(t))+\Phi(h, t) \dot{u}(t)] \mathrm{d} t=h \int_{0}^{1} \Phi(h, c h)[\dot{u}(c h)-f(u(c h))] \mathrm{d} c \\
= & h \sum_{i=0}^{s-1} \underbrace{\int_{0}^{1} P_{i}(c) \Phi(h, c h) \mathrm{d} c}_{=O\left(h^{i}\right)} \underbrace{\left[\hat{\Gamma}_{i}-\Gamma_{i}(u)\right]}_{=O\left(h^{2 s-i}\right)}-h \sum_{i \geq s} \underbrace{\int_{0}^{1} P_{i}(c) \Phi(h, c h)}_{=O\left(h^{i}\right)} \mathrm{d} \underbrace{\Gamma_{i}(u)}_{O\left(h^{i}\right)}=O\left(h^{2 s+1}\right) .
\end{aligned}
$$

Remark 3 From the result of the last two theorems, one deduces that a $\operatorname{LIM}\left(k_{1}, k_{2}, s\right)$ method:

- has order $2 s$, provided that $k_{1}, k_{2} \geq s$;

- is energy-conserving, when the Hamiltonian $H$ in (9) is a polynomial of degree not larger that $2 k_{2} / s$. 
We observe that, even when $H$ is not a polynomial, a practical energy conservation can always be gained by choosing $k_{2}$ large enough, so that the $O\left(h^{2 k_{2}+1}\right)$ energy error falls below the round-off error level: for this reason, in the sequel we shall make no distinction between the two cases.

Let us now derive a compact formulation of the discrete problem generated by the method. As is clear, it is enough to compute the coefficients $\hat{\Gamma}_{i}, i=0, \ldots, s-1$, of the polynomial approximation (32). Let us then define the following vectors and matrices:

$$
\begin{aligned}
& \boldsymbol{\Gamma}=\left(\begin{array}{c}
\hat{\Gamma}_{0} \\
\vdots \\
\hat{\Gamma}_{s-1}
\end{array}\right), \quad \boldsymbol{\rho}=\left(\begin{array}{ccc}
\hat{\rho}_{00} & \ldots & \hat{\rho}_{0, s-1} \\
\vdots & & \vdots \\
\hat{\rho}_{s-1,0} & \ldots & \hat{\rho}_{s-1, s-1}
\end{array}\right), \quad \boldsymbol{\gamma}=\left(\begin{array}{c}
\hat{\gamma}_{0} \\
\vdots \\
\hat{\gamma}_{s-1}
\end{array}\right) \\
& \hat{\boldsymbol{c}}=\left(\begin{array}{c}
\hat{c}_{1} \\
\vdots \\
\hat{c}_{k_{1}}
\end{array}\right), \quad \boldsymbol{c}=\left(\begin{array}{c}
c_{1} \\
\vdots \\
c_{k_{2}}
\end{array}\right), \quad u(\hat{\boldsymbol{c}} h)=\left(\begin{array}{c}
u\left(\hat{c}_{1} h\right) \\
\vdots \\
u\left(\hat{c}_{k_{1}} h\right)
\end{array}\right), \quad u(\boldsymbol{c} h)=\left(\begin{array}{c}
u\left(c_{1} h\right) \\
\vdots \\
u\left(c_{k_{2}} h\right)
\end{array}\right), \\
& S(u(\hat{\boldsymbol{c}} h))=\left(\begin{array}{ccc}
S\left(u\left(\hat{c}_{1} h\right)\right) & & \\
& \ddots & \\
& & S\left(u\left(\hat{c}_{k_{1}} h\right)\right)
\end{array}\right), \quad \nabla H(u(\boldsymbol{c} h))=\left(\begin{array}{c}
\nabla H\left(u\left(c_{1} h\right)\right) \\
\vdots \\
\nabla H\left(u\left(c_{k_{2}} h\right)\right)
\end{array}\right), \\
& \hat{\mathcal{P}}_{s}=\left(P_{j-1}\left(\hat{c}_{i}\right)\right), \hat{\mathcal{I}}_{s}=\left(\int_{0}^{\hat{c}_{i}} P_{j-1}(x) \mathrm{d} x\right) \in \mathbb{R}^{k_{1} \times s}, \quad \hat{\Omega}=\operatorname{diag}\left(\hat{b}_{1}, \ldots, \hat{b}_{k_{1}}\right), \\
& \mathcal{P}_{s}=\left(P_{j-1}\left(c_{i}\right)\right), \mathcal{I}_{s}=\left(\int_{0}^{c_{i}} P_{j-1}(x) \mathrm{d} x\right) \in \mathbb{R}^{k_{2} \times s}, \quad \Omega=\operatorname{diag}\left(b_{1}, \ldots, b_{k_{2}}\right),
\end{aligned}
$$

and

$$
\hat{\mathbf{1}}=\left(\begin{array}{c}
1 \\
\vdots \\
1
\end{array}\right) \in \mathbb{R}^{k_{1}}, \quad \mathbf{1}=\left(\begin{array}{c}
1 \\
\vdots \\
1
\end{array}\right) \in \mathbb{R}^{k_{2}} .
$$

Moreover, hereafter we set $I$ the identity matrix having the same dimension as that of the state vector. One has, then:

$$
\begin{aligned}
u(\hat{\boldsymbol{c}} h) & =\hat{\mathbf{1}} \otimes y_{0}+h \hat{\mathcal{I}}_{s} \otimes I \boldsymbol{\Gamma}, \\
u(\boldsymbol{c} h) & =\mathbf{1} \otimes y_{0}+h \mathcal{I}_{s} \otimes I \boldsymbol{\Gamma}, \\
\boldsymbol{\gamma} & =\left[\mathcal{P}_{s}^{\top} \Omega \otimes I\right] \nabla H\left(\mathbf{1} \otimes y_{0}+h \mathcal{I}_{s} \otimes I \boldsymbol{\Gamma}\right), \\
\boldsymbol{\rho} & =\left[\hat{\mathcal{P}}_{s}^{\top} \hat{\Omega} \otimes I\right] S\left(\hat{\mathbf{1}} \otimes y_{0}+h \hat{\mathcal{I}}_{s} \otimes I \boldsymbol{\Gamma}\right)\left[\hat{\mathcal{P}}_{s} \otimes I\right], \\
\boldsymbol{\Gamma} & =\boldsymbol{\rho} \gamma
\end{aligned}
$$

Consequently, one obtains the following discrete problem involving only $\boldsymbol{\Gamma}$ :

$$
G(\boldsymbol{\Gamma}):=\boldsymbol{\Gamma}-\left[\hat{\mathcal{P}}_{s}^{\top} \hat{\Omega} \otimes I\right] S\left(\hat{\mathbf{1}} \otimes y_{0}+h \hat{\mathcal{I}}_{s} \otimes I \boldsymbol{\Gamma}\right)\left[\hat{\mathcal{P}}_{s} \mathcal{P}_{s}^{\top} \Omega \otimes I\right] \nabla H\left(\mathbf{1} \otimes y_{0}+h \mathcal{I}_{s} \otimes I \boldsymbol{\Gamma}\right)=\mathbf{0} .
$$


Remark 4 We observe that, in the case $k_{1}=s$, we obtain the methods defined in [5] (compare [43) with [5, Eq. (36)]) which, in turn, are akin to those in [22]. Their derivation, however, is now done within a different framework, yielding more general methods (i.e., using $k_{1}>s$ ), though having the same order of accuracy. Moreover, the line integral approach used to derive (43) allows us to easily extend a technique devised for the efficient implementation of line integral methods applied to Hamiltonian problems (see e.g. [14]). This aspect will be faced in the next section.

Another important property of the $\operatorname{LIM}\left(k_{1}, k_{2}, s\right)$ method is symmetry, namely if we apply the method to the equation in (9), starting from $y_{1}$ as defined in (33) and using a stepsize $-h$, this brings us back to $y_{0}$. To prove this property, we need the following preliminary result.

Lemma 6 With reference to (40)-42, let us define the following matrices:

$$
I_{\ell}=\left(\begin{array}{ccc}
1 & & \\
& \ddots & \\
& & 1
\end{array}\right), D_{\ell}=\left(\begin{array}{ccc}
(-1)^{0} & & \\
& \ddots & \\
& & (-1)^{\ell-1}
\end{array}\right), P_{\ell}=\left(\begin{array}{lll} 
& \\
& . & \\
& &
\end{array}\right) \in \mathbb{R}^{\ell \times \ell}
$$

and set $\boldsymbol{e}_{1} \in \mathbb{R}^{s}$ the first unit vector. Then,

$$
\begin{aligned}
& D_{\ell}^{2}=P_{\ell}^{2}=I_{\ell}, \quad P_{k_{1}} \hat{\mathcal{I}}_{s} D_{s}=\hat{\mathbf{1}} \boldsymbol{e}_{1}^{\top}-\hat{\mathcal{I}}_{s}, \quad P_{k_{2}} \mathcal{I}_{s} D_{s}=\mathbf{1} \boldsymbol{e}_{1}^{\top}-\mathcal{I}_{s}, \\
& P_{k_{1}} \hat{\mathcal{P}}_{s} D_{s}=\hat{\mathcal{P}}_{s}, \quad P_{k_{2}} \mathcal{P}_{s} D_{s}=\mathcal{P}_{s}, \quad P_{k_{1}} \hat{\Omega} P_{k_{1}}=\hat{\Omega}, \quad P_{k_{2}} \Omega P_{k_{2}}=\Omega .
\end{aligned}
$$

Proof See [21, Lemma 3]. In particular all the properties, but the first one, derive from the symmetry of the abscissae (and, then, of the weights) 28)-29.

We are now in the position of proving the symmetry of the methods.

Theorem 5 For all $k_{1}, k_{2} \geq s$, the method $\operatorname{LIM}\left(k_{1}, k_{2}, s\right)$ is symmetric.

Proof By using the method on the equation (9) starting at $y_{1}$, as defined in (33), with timestep $-h$ we obtain (see $(43 p)$

$$
\overline{\boldsymbol{\Gamma}} \equiv\left(\begin{array}{c}
\bar{\Gamma}_{0} \\
\vdots \\
\bar{\Gamma}_{s-1}
\end{array}\right)=\left[\hat{\mathcal{P}}_{s}^{\top} \hat{\Omega} \otimes I\right] S\left(\hat{\mathbf{1}} \otimes y_{1}-h \hat{\mathcal{I}}_{s} \otimes I \overline{\mathbf{\Gamma}}\right)\left[\hat{\mathcal{P}}_{s} \mathcal{P}_{s}^{\top} \Omega \otimes I\right] \nabla H\left(\mathbf{1} \otimes y_{1}-h \mathcal{I}_{s} \otimes I \overline{\boldsymbol{\Gamma}}\right),
$$

with the new approximation given by $\bar{y}_{0}=y_{1}-h \bar{\Gamma}_{0}$. We have then to show that $\bar{y}_{0}=y_{0}$. This will follow from the fact that (see (44))

$$
\bar{\Gamma}_{j}=(-1)^{j} \hat{\Gamma}_{j}=: \Gamma_{j}^{*}, \quad j=0, \ldots, s-1, \quad \Longleftrightarrow \quad \overline{\boldsymbol{\Gamma}}=D_{s} \otimes I \boldsymbol{\Gamma}=:\left(\begin{array}{c}
\Gamma_{0}^{*} \\
\vdots \\
\Gamma_{s-1}^{*}
\end{array}\right) \equiv \boldsymbol{\Gamma}^{*} .
$$

We shall prove this statement by showing that $\boldsymbol{\Gamma}^{*}$ satisfies the same equation 45 which implicitly defines $\overline{\boldsymbol{\Gamma}}$. One has: 


$$
\begin{aligned}
\boldsymbol{\Gamma}^{*} & =D_{s} \otimes I \boldsymbol{\Gamma} \\
& =\left[D_{s} \hat{\mathcal{P}}_{s}^{\top} \hat{\Omega} \otimes I\right] S\left(\hat{\mathbf{1}} \otimes y_{0}+h \hat{\mathcal{I}}_{s} \otimes I \boldsymbol{\Gamma}\right)\left[\hat{\mathcal{P}}_{s} \mathcal{P}_{s}^{\top} \Omega \otimes I\right] \nabla H\left(\mathbf{1} \otimes y_{0}+h \mathcal{I}_{s} \otimes I \boldsymbol{\Gamma}\right) \\
& =\left[\hat{\mathcal{P}}_{s}^{\top} \hat{\Omega} P_{k_{1}} \otimes I\right] S\left(\hat{\mathbf{1}} \otimes y_{0}+h \hat{\mathcal{I}}_{s} \otimes I \boldsymbol{\Gamma}\right)\left[P_{k_{1}}^{2} \hat{\mathcal{P}}_{s} \mathcal{P}_{s}^{\top} \Omega \otimes I\right] \nabla H\left(\mathbf{1} \otimes y_{0}+h \mathcal{I}_{s} \otimes I \boldsymbol{\Gamma}\right) \\
& =\left[\hat{\mathcal{P}}_{s}^{\top} \hat{\Omega} \otimes I\right] S\left(P_{k_{1}} \hat{\mathbf{1}} \otimes y_{0}+h P_{k_{1}} \hat{\mathcal{I}}_{s} D_{s}^{2} \otimes I \boldsymbol{\Gamma}\right)\left[P_{k_{1}} \hat{\mathcal{P}}_{s} D_{s}^{2} \mathcal{P}_{s}^{\top} \Omega \otimes I\right] \nabla H\left(\mathbf{1} \otimes y_{0}+h \mathcal{I}_{s} \otimes I \boldsymbol{\Gamma}\right) \\
& =\left[\hat{\mathcal{P}}_{s}^{\top} \hat{\Omega} \otimes I\right] S\left(\hat{\mathbf{1}} \otimes y_{0}+h\left(\hat{\mathbf{1}} \boldsymbol{e}_{1}^{\top}-\hat{\mathcal{I}}_{s}\right) \otimes I \boldsymbol{\Gamma}^{*}\right)\left[\hat{\mathcal{P}}_{s} \mathcal{P}_{s}^{\top} \Omega P_{k_{2}} \otimes I\right] \nabla H\left(\mathbf{1} \otimes y_{0}+h \mathcal{I}_{s} \otimes I \boldsymbol{\Gamma}\right) \\
& =\left[\hat{\mathcal{P}}_{s}^{\top} \hat{\Omega} \otimes I\right] S\left(\hat{\mathbf{1}} \otimes\left(y_{0}+h \hat{\Gamma}_{0}\right)-h \hat{\mathcal{I}}_{s} \otimes I \boldsymbol{\Gamma}^{*}\right)\left[\hat{\mathcal{P}}_{s} \mathcal{P}_{s}^{\top} \Omega \otimes I\right] \nabla H\left(P_{k_{2}} \mathbf{1} \otimes y_{0}+h P_{k_{2}} \mathcal{I}_{s} D_{s}^{2} \otimes I \mathbf{\Gamma}\right) \\
& =\left[\hat{\mathcal{P}}_{s}^{\top} \hat{\Omega} \otimes I\right] S\left(\hat{\mathbf{1}} \otimes y_{1}-h \hat{\mathcal{I}}_{s} \otimes I \boldsymbol{\Gamma}^{*}\right)\left[\hat{\mathcal{P}}_{s} \mathcal{P}_{s}^{\top} \Omega \otimes I\right] \nabla H\left(\mathbf{1} \otimes y_{0}+h\left(\mathbf{1} \boldsymbol{e}_{1}^{\top}-\mathcal{I}_{s}\right) \otimes I \boldsymbol{\Gamma}^{*}\right) \\
& =\left[\hat{\mathcal{P}}_{s}^{\top} \hat{\Omega} \otimes I\right] S\left(\hat{\mathbf{1}} \otimes y_{1}-h \hat{\mathcal{I}}_{s} \otimes I \boldsymbol{\Gamma}^{*}\right)\left[\hat{\mathcal{P}}_{s} \mathcal{P}_{s}^{\top} \Omega \otimes I\right] \nabla H\left(\mathbf{1} \otimes\left(y_{0}+h \hat{\Gamma}_{0}\right)-h \mathcal{I}_{s} \otimes I \boldsymbol{\Gamma}^{*}\right) \\
& =\left[\hat{\mathcal{P}}_{s}^{\top} \hat{\Omega} \otimes I\right] S\left(\hat{\mathbf{1}} \otimes y_{1}-h \hat{\mathcal{I}}_{s} \otimes I \boldsymbol{\Gamma}^{*}\right)\left[\hat{\mathcal{P}}_{s} \mathcal{P}_{s}^{\top} \Omega \otimes I\right] \nabla H\left(\mathbf{1} \otimes y_{1}-h \mathcal{I}_{s} \otimes I \boldsymbol{\Gamma}^{*}\right) .
\end{aligned}
$$

Consequently, the statement follows.

\subsection{Solving the discrete problem}

Preliminarily, let us recall that, by the properties of Legendre polynomials (see, e.g., [8]), one has:

$$
\hat{\mathcal{P}}_{s}^{\top} \hat{\Omega} \hat{\mathcal{I}}_{s}=\mathcal{P}_{s}^{\top} \Omega \mathcal{I}_{s}=X_{s} \equiv\left(\begin{array}{cccc}
\xi_{0} & -\xi_{1} & & \\
\xi_{1} & 0 & \ddots & \\
& \ddots & \ddots & -\xi_{s-1} \\
& & \xi_{s-1} & 0
\end{array}\right), \quad \xi_{i}=\frac{1}{2 \sqrt{\left|4 i^{2}-1\right|}},
$$

and

$$
\hat{\mathcal{P}}_{s}^{\top} \hat{\Omega} \hat{\mathcal{P}}_{s}=I_{s}, \quad \mathcal{P}_{s}^{\top} \Omega \mathbf{1}=\boldsymbol{e}_{1} .
$$

Next, we observe that the discrete problem (43) naturally induces the fixed-point iteration:

$$
\boldsymbol{\Gamma}^{\ell+1}=\left[\hat{\mathcal{P}}_{s}^{\top} \hat{\Omega} \otimes I\right] S\left(\hat{\mathbf{1}} \otimes y_{0}+h \hat{\mathcal{I}}_{s} \otimes I \boldsymbol{\Gamma}^{\ell}\right)\left[\hat{\mathcal{P}}_{s} \mathcal{P}_{s}^{\top} \Omega \otimes I\right] \nabla H\left(\mathbf{1} \otimes y_{0}+h \mathcal{I}_{s} \otimes I \boldsymbol{\Gamma}^{\ell}\right), \quad \ell=0,1, \ldots
$$

The following theorem provides sufficient condition for its convergence.

Theorem 6 Assume that the function $S(y)$ and $\nabla H(y)$ at the right-hand side in (9) are Lipschitz with constant $L$ in a suitable closed ball of radius $\rho$ centered at $y_{0}$. Let us denote

$$
\alpha_{0}=\max _{y}\left\{\|S(y)\|:\left\|y-y_{0}\right\| \leq \rho\right\}, \quad \alpha_{1}=\max _{y}\left\{\|\nabla H(y)\|:\left\|y-y_{0}\right\| \leq \rho\right\}, \quad \alpha=\max \left\{\alpha_{0}, \alpha_{1}\right\},
$$

where, hereafter, $\|\cdot\|$ denotes the infinity norm. Moreover, let us set:

$$
\rho_{1}=\left\|\hat{\mathcal{P}}_{s}^{\top} \hat{\Omega}\right\| \cdot\left\|\hat{\mathcal{P}}_{s}\right\| \cdot\left\|\mathcal{P}_{s}^{\top} \Omega\right\|, \quad \rho_{2}=\left\|\hat{\mathcal{I}}_{s}\right\|+\left\|\mathcal{I}_{s}\right\| .
$$

Then, starting at $\boldsymbol{\Gamma}^{0}=\mathbf{0}$, the iteration (48) converges, provided that the timestep $h$ satisfies

$$
0 \leq h<h^{*}:=\min \left\{\frac{1}{\rho_{1} \rho_{2} \alpha L}, \frac{\rho}{\rho_{2}\left\|S\left(y_{0}\right) \nabla H\left(y_{0}\right)\right\|}\right\} .
$$


Proof Starting from $\boldsymbol{\Gamma}^{0}=\mathbf{0}$, by virtue of 47 one obtains $\boldsymbol{\Gamma}^{1}=\boldsymbol{e}_{1} \otimes S\left(y_{0}\right) \nabla H\left(y_{0}\right)$. Consequently, from 49 it follows that

$$
\left\|h \hat{\mathcal{I}}_{s} \otimes I \Gamma^{1}\right\|,\left\|h \mathcal{I}_{s} \otimes I \Gamma^{1}\right\|<\rho .
$$

Next, assume that

$$
\left\|h \hat{\mathcal{I}}_{s} \otimes I \Gamma^{i}\right\|,\left\|h \mathcal{I}_{s} \otimes I \Gamma^{i}\right\|<\rho, \quad i=\ell-1, \ell .
$$

Then,

$$
\begin{aligned}
\left\|\boldsymbol{\Gamma}^{\ell+1}-\boldsymbol{\Gamma}^{\ell}\right\|= & \left.\| \hat{\mathcal{P}}_{s}^{\top} \hat{\Omega} \otimes I\right] S\left(\hat{\mathbf{1}} \otimes y_{0}+h \hat{\mathcal{I}}_{s} \otimes I \boldsymbol{\Gamma}^{\ell}\right)\left[\hat{\mathcal{P}}_{s} \mathcal{P}_{s}^{\top} \Omega \otimes I\right] \nabla H\left(\mathbf{1} \otimes y_{0}+h \mathcal{I}_{s} \otimes I \boldsymbol{\Gamma}^{\ell}\right) \\
& -\left[\hat{\mathcal{P}}_{s}^{\top} \hat{\Omega} \otimes I\right] S\left(\hat{\mathbf{1}} \otimes y_{0}+h \hat{\mathcal{I}}_{s} \otimes I \boldsymbol{\Gamma}^{\ell-1}\right)\left[\hat{\mathcal{P}}_{s} \mathcal{P}_{s}^{\top} \Omega \otimes I\right] \nabla H\left(\mathbf{1} \otimes y_{0}+h \mathcal{I}_{s} \otimes I \boldsymbol{\Gamma}^{\ell-1}\right) \| \\
= & \|\left[\hat{\mathcal{P}}_{s}^{\top} \hat{\Omega} \otimes I\right] S\left(\hat{\mathbf{1}} \otimes y_{0}+h \hat{\mathcal{I}}_{s} \otimes I \boldsymbol{\Gamma}^{\ell}\right)\left[\hat{\mathcal{P}}_{s} \mathcal{P}_{s}^{\top} \Omega \otimes I\right] \nabla H\left(\mathbf{1} \otimes y_{0}+h \mathcal{I}_{s} \otimes I \boldsymbol{\Gamma}^{\ell}\right) \\
& \pm\left[\hat{\mathcal{P}}_{s}^{\top} \hat{\Omega} \otimes I\right] S\left(\hat{\mathbf{1}} \otimes y_{0}+h \hat{\mathcal{I}}_{s} \otimes I \boldsymbol{\Gamma}^{\ell}\right)\left[\hat{\mathcal{P}}_{s} \mathcal{P}_{s}^{\top} \Omega \otimes I\right] \nabla H\left(\mathbf{1} \otimes y_{0}+h \mathcal{I}_{s} \otimes I \boldsymbol{\Gamma}^{\ell-1}\right) \\
& -\left[\hat{\mathcal{P}}_{s}^{\top} \hat{\Omega} \otimes I\right] S\left(\hat{\mathbf{1}} \otimes y_{0}+h \hat{\mathcal{I}}_{s} \otimes I \boldsymbol{\Gamma}^{\ell-1}\right)\left[\hat{\mathcal{P}}_{s} \mathcal{P}_{s}^{\top} \Omega \otimes I\right] \nabla H\left(\mathbf{1} \otimes y_{0}+h \mathcal{I}_{s} \otimes I \boldsymbol{\Gamma}^{\ell-1}\right) \| \\
\leq & h \rho_{1} \rho_{2} \alpha L\left\|\boldsymbol{\Gamma}^{\ell}-\boldsymbol{\Gamma}^{\ell-1}\right\| .
\end{aligned}
$$

Consequently, we have a contraction, for all $h<h^{*}$.

As is clear from (49), when the right-hand side in (9) has a large linear part, the fixed-point iteration (48) may require a quite small timestep to converge. When this happens, a Newton-type iteration for solving (43) would be more appropriate, which we now sketch below.

To begin with, we observe that, when $k_{1}=k_{2}=s$, then $\hat{\mathcal{P}}_{s}=\mathcal{P}_{s}, \hat{\mathcal{I}}_{s}=\mathcal{I}_{s}, \hat{\Omega}=\Omega$, which are $s \times s$ matrices, and $\mathcal{P}_{s}^{-1}=\mathcal{P}_{s}^{\top} \Omega$ (see (47)). In such a case, (43) becomes (using the notation in (9p)

$$
G(\boldsymbol{\Gamma}):=\boldsymbol{\Gamma}-\mathcal{P}_{s}^{\top} \Omega \otimes I f\left(\mathbf{1} \otimes y_{0}+h \mathcal{I}_{s} \otimes I \boldsymbol{\Gamma}\right)=\mathbf{0} .
$$

The use of the simplified Newton iteration for solving such an equation then becomes, by virtue of 46):

$$
\begin{aligned}
\text { solve: } & {\left[I_{s} \otimes I-h X_{s} \otimes f^{\prime}\left(y_{0}\right)\right] \Delta^{\ell}=-G\left(\boldsymbol{\Gamma}^{\ell}\right) } \\
\text { set: } & \boldsymbol{\Gamma}^{\ell+1}=\boldsymbol{\Gamma}^{\ell}+\Delta^{\ell}, \quad \ell=0,1, \ldots,
\end{aligned}
$$

where, as is usual, $f^{\prime}\left(y_{0}\right)$ denotes the Jacobian of $f(y)$ evaluated at $y_{0}$. Also in this case, the initial guess $\boldsymbol{\Gamma}^{0}=\mathbf{0}$ can be conveniently used. However, since the dimension of the discrete problem is always $s$, whichever are $k_{1}$ and $k_{2}$, we shall continue using the iteration (50) even when $k_{1}>s$ and/or $k_{2}>s$, so that $G\left(\boldsymbol{\Gamma}^{\ell}\right)$ is now evaluated according to 43$)$. In any case, the straight solution of the linear systems in 50 would require the factorization of a matrix whose dimension is $s$ times larger than that of the continuous problem (9). In order to obtain a comparably effective, though less expensive, Newton-type iteration, we shall consider a corresponding Newton-splitting blended iteration. This iteration, at first devised in [4, has been studied in [16] (see also [19]), and implemented in the computational codes BiM 17 and BiMD [18, respectively solving stiff ODE-IVPs and linearly implicit DAEs. Later on, it has been considered for HBVMs [14, 6, 20, as well as for 
other line integral methods (see, e.g., 21]). We here only sketch the final iteration ${ }^{6}$

$$
\begin{aligned}
\text { set: } & \rho_{s}=\min _{\lambda \in \sigma\left(X_{s}\right)}|\lambda|, \quad \Theta=\left[I-h \rho_{s} f^{\prime}\left(y_{0}\right)\right]^{-1}, \quad \boldsymbol{\Gamma}^{0}=\mathbf{0} \\
\text { for } \ell=0,1, \ldots: & \boldsymbol{\eta}^{\ell}=-G\left(\boldsymbol{\Gamma}^{\ell}\right), \quad \boldsymbol{\eta}_{1}^{\ell}=\left[\rho_{s} X_{s}^{-1}\right] \otimes I \boldsymbol{\eta}^{\ell} \\
& \boldsymbol{\Gamma}^{\ell+1}=\boldsymbol{\Gamma}^{\ell}-I_{s} \otimes \Theta\left[\boldsymbol{\eta}_{1}^{\ell}+I_{s} \otimes(I-\Theta)\left(\boldsymbol{\eta}^{\ell}-\boldsymbol{\eta}_{1}^{\ell}\right)\right]
\end{aligned}
$$

Consequently, the iteration (51) now requires only the factorization of one matrix (i.e., $\Theta^{-1}$ ), having the same size as that of the continuous problem.

\section{$4 \quad$ Numerical tests}

We here report a few numerical tests describing particular instances of the gyrocenter dynamics of a charged particle [30, i.e. :

1. the case of a dipole magnetic field with zero electric potential,

2. the case of a tokamak magnetic field with zero electric potential,

3. the case of a dipole magnetic field with quadratic electric field.

In the first two problems, the electric potential $\phi(x)$ is assumed to be 0 , which is called the temporal gauge. Consequently, the magnetic vector potential (2) is enough to completely define the problem, along with the constant $\mu$ and the initial conditions. All numerical tests have been done on an Intel i7 computer with 16GB of memory, running Matlab 2019a.

In the first two problems, the fixed-point iteration (48) is used for solving the generated discrete problems, since the linear part of the corresponding right-hand sides turns out to be very small (of the order of unity), so that there is no gain in using the blended iteration (51), whose computational cost per iterate is higher. However, in the third test problem the blended iteration turns out to have a superior performance. Moreover, in the numerical tests we set $k_{1}=s$ and $k_{2} \equiv k \geq s$, so that all $\operatorname{LIM}\left(s, k_{2}, s\right)$ methods have order $2 s$.

Dipole magnetic field. The dipole magnetic field has wide applications in different branches of physics, underlying many examples of cosmic magnetic fields, such as the earth magnetic field and the neutron stars magnetic field. The vector potential is

$$
A(x)=\frac{M}{\rho^{3}}\left(x_{2}, \quad-x_{1}, \quad 0\right)^{\top}, \quad \rho=\|x\|,
$$

\footnotetext{
${ }^{6}$ The parameter $\rho_{s}$ is determined according to a linear analysis of convergence [16, 19] and, as usual, $\sigma\left(X_{s}\right)$ denotes the spectrum of matrix $X_{s}$.
} 
Table 1: Maximum Hamiltonian error when solving the dipole magnetic field problem on the interval $\left[0,10^{3}\right]$, by using $\operatorname{LIM}(s, k, s)$ with timestep $h=0.4$.

\begin{tabular}{|r|rrrrr|}
\hline & \multicolumn{5}{|c|}{$s$} \\
\hline$k$ & 1 & 2 & 3 & 4 & 5 \\
\hline 1 & $2.689 \mathrm{e}-02$ & & & & \\
2 & $6.163 \mathrm{e}-04$ & $5.103 \mathrm{e}-03$ & & & \\
3 & $3.549 \mathrm{e}-06$ & $5.551 \mathrm{e}-05$ & $2.785 \mathrm{e}-04$ & & \\
4 & $8.366 \mathrm{e}-08$ & $6.909 \mathrm{e}-07$ & $8.613 \mathrm{e}-06$ & $1.374 \mathrm{e}-05$ & \\
5 & $1.425 \mathrm{e}-09$ & $1.371 \mathrm{e}-08$ & $1.040 \mathrm{e}-07$ & $3.796 \mathrm{e}-07$ & $6.394 \mathrm{e}-07$ \\
6 & $3.256 \mathrm{e}-11$ & $4.590 \mathrm{e}-10$ & $1.998 \mathrm{e}-09$ & $7.869 \mathrm{e}-09$ & $1.552 \mathrm{e}-08$ \\
7 & $1.776 \mathrm{e}-15$ & $8.698 \mathrm{e}-12$ & $5.307 \mathrm{e}-11$ & $1.455 \mathrm{e}-10$ & $2.828 \mathrm{e}-10$ \\
8 & $2.220 \mathrm{e}-15$ & $1.776 \mathrm{e}-15$ & $5.653 \mathrm{e}-13$ & $2.850 \mathrm{e}-12$ & $4.602 \mathrm{e}-12$ \\
9 & & $1.776 \mathrm{e}-15$ & $1.776 \mathrm{e}-15$ & $1.776 \mathrm{e}-15$ & $1.776 \mathrm{e}-15$ \\
10 & & & $2.220 \mathrm{e}-15$ & $1.776 \mathrm{e}-15$ & $1.776 \mathrm{e}-15$ \\
\hline
\end{tabular}

Table 2: Dipole magnetic field problem solved on the interval $[0,40]$ with timestep $h$ (times in sec).

\begin{tabular}{|c|c|c|c|c|c|c|c|c|c|c|c|c|c|c|c|}
\hline & \multicolumn{3}{|c|}{$\operatorname{LIM}(1,7,1)$} & \multicolumn{3}{|c|}{$\operatorname{LIM}(2,8,2)$} & \multicolumn{3}{|c|}{$\operatorname{LIM}(3,9,3)$} & \multicolumn{3}{|c|}{$\operatorname{LIM}(4,9,4)$} & \multicolumn{3}{|c|}{$\operatorname{LIM}(5,9,5)$} \\
\hline$h$ & err & rate & time & err & rate & time & err & rate & time & err & rate & time & err & rate & time \\
\hline 0.4 & $1.05 \mathrm{e} 00$ & - & 0.2 & $1.58 \mathrm{e}-02$ & - & 0.3 & $1.82 \mathrm{e}-03$ & - & 0.2 & $4.12 \mathrm{e}-05$ & - & 0.2 & $1.78 \mathrm{e}-07$ & - & 0.2 \\
\hline $2^{-1} 0.4$ & $2.90 \mathrm{e}-01$ & 1.9 & 0.3 & $1.71 \mathrm{e}-03$ & 3.2 & 0.4 & $3.35 \mathrm{e}-05$ & 5.8 & 0.4 & $9.44 \mathrm{e}-08$ & 8.8 & 0.3 & $1.68 \mathrm{e}-09$ & 6.7 & 0.3 \\
\hline $2^{-2} 0.4$ & $7.44 \mathrm{e}-02$ & 2.0 & 0.4 & $1.20 \mathrm{e}-04$ & 3.8 & 0.6 & $5.16 \mathrm{e}-07$ & 6.0 & 0.6 & $4.74 \mathrm{e}-10$ & 7.6 & 0.5 & $1.11 \mathrm{e}-12$ & 10.6 & 0.5 \\
\hline $2^{-3} 0.4$ & $1.87 \mathrm{e}-02$ & 2.0 & 0.6 & $7.69 \mathrm{e}-06$ & 4.0 & 0.9 & $8.06 \mathrm{e}-09$ & 6.0 & 1.0 & $2.18 \mathrm{e}-12$ & 7.8 & 0.9 & $4.00 \mathrm{e}-13$ & $* *$ & 0.9 \\
\hline $2^{-4} 0.4$ & $4.68 \mathrm{e}-03$ & 2.0 & 1.0 & $4.84 \mathrm{e}-07$ & 4.0 & 1.6 & $1.26 \mathrm{e}-10$ & 6.0 & 1.6 & $3.32 \mathrm{e}-13$ & $* *$ & 1.6 & & & \\
\hline $2^{-5} 0.4$ & $1.17 \mathrm{e}-03$ & 2.0 & 1.8 & $3.03 \mathrm{e}-08$ & 4.0 & 2.8 & $1.54 \mathrm{e}-12$ & 6.4 & 3.0 & & & & & & \\
\hline $2^{-6} 0.4$ & $2.93 \mathrm{e}-04$ & 2.0 & 3.1 & $1.89 \mathrm{e}-09$ & 4.0 & 5.0 & $1.34 \mathrm{e}-13$ & $* *$ & 5.4 & & & & & & \\
\hline $2^{-7} 0.4$ & $7.32 \mathrm{e}-05$ & 2.0 & 5.8 & $1.19 \mathrm{e}-10$ & 4.0 & 9.2 & & & & & & & & & \\
\hline $2^{-8} 0.4$ & $1.83 \mathrm{e}-05$ & 2.0 & 10.5 & $7.02 \mathrm{e}-12$ & 4.1 & 17.0 & & & & & & & & & \\
\hline $2^{-9} 0.4$ & $4.58 \mathrm{e}-06$ & 2.0 & 20.0 & $7.91 \mathrm{e}-13$ & $* *$ & 32.9 & & & & & & & & & \\
\hline $2^{-10} 0.4$ & $1.14 \mathrm{e}-06$ & 2.0 & 37.5 & & & & & & & & & & & & \\
\hline $2^{-11} 0.4$ & $2.86 \mathrm{e}-07$ & 2.0 & 68.2 & & & & & & & & & & & & \\
\hline
\end{tabular}

with $M$ being the dipole moment, which can be either positive or negative $]^{7}$ Consequently, one obtains:

$$
\begin{aligned}
B(x) & =-\frac{M}{\rho^{5}}\left(3 x_{1} x_{3}, \quad 3 x_{2} x_{3}, \quad 2 x_{3}^{2}-x_{1}^{2}-x_{2}^{2}\right)^{\top}, \\
\|B(x)\| & =|M| \frac{\sqrt{\rho^{2}+3 x_{3}^{2}}}{\rho^{4}} \\
b(x) & =\frac{-M /|M|}{\rho \sqrt{\rho^{2}+3 x_{3}^{2}}}\left(\begin{array}{lll}
3 x_{1} x_{3}, & 3 x_{2} x_{3}, & 2 x_{3}^{2}-x_{1}^{2}-x_{2}^{2}
\end{array}\right)^{\top} .
\end{aligned}
$$

In the numerical tests, we set

$$
M=10^{3}, \quad \mu=10^{-2}, \quad y(0)=(1,1,1,0.01)^{\top} .
$$

\footnotetext{
${ }^{7}$ In the case of earth, the constant $M=-8 \times 10^{15}$.
} 

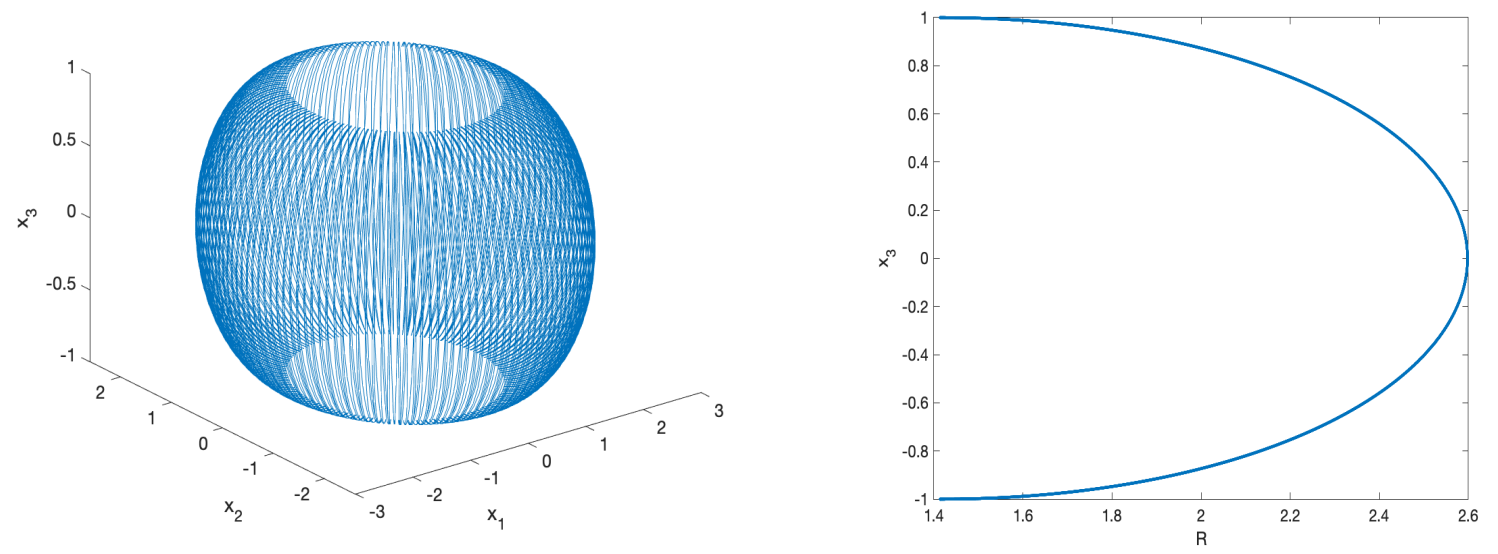

Figure 1: Dipole magnetic problem. Left plot: solution trajectory in the phase space; right plot: solution in the coordinates $(55)$.

In Figure 1 we show the 3D trajectory of the gyrocenter (left plot), and its $2 \mathrm{D}$ representation (right plot) in the coordinates

$$
R=\sqrt{x_{1}^{2}+x_{2}^{2}}, \quad x_{3},
$$

for $t \in\left[0,10^{3}\right]$.

At first, in Table 1 we show the maximum Hamiltonian error, by using a timestep $h=0.4$, for the $\operatorname{LIM}(s, k, s)$ methods, $s=1, \ldots, 5$, and $k=s, \ldots, 10$. As one may expect, as the value of $k$ increases, the Hamiltonian error decreases. In particular, in order to obtain energy-conservation up to round-off error level, a value $k=7$ is enough when $s=1, k=8$ is sufficient in the case $s=2$, whereas $k=9$ is appropriately chosen, for $s=3,4,5$.

Then, in Table 2, we list the solution error, along with the estimate convergence rate, for the $\operatorname{LIM}(1,7,1), \operatorname{LIM}(2,8,2)$, and $\operatorname{LIM}(s, 9, s), s=3,4,5$, methods when using a timestep $h=2^{-i} 0.4$, $i \geq 0$, to cover the interval $[0,40]$. We also list the corresponding execution times (in sec). As one may see, the convergence order $2 s$ is satisfied for all methods. Moreover, the higher-order methods turns out to be much more efficient than the lower-order ones. We notice that the $\operatorname{LIM}(1,7,1)$, which is the lowest-order method, is equivalent to the method used in [28].

Tokamak magnetic field. The tokamak magnetic field is used to confine a hot plasma in the shape of a torus. The axisymmetric tokamak geometry is illustrated in Figure2, where $R$ is defined according to (55), $r=\sqrt{\left(R-R_{0}\right)^{2}+x_{3}^{2}}$, and $R_{0}$ is the main radius. We give an example for the vector potential

$$
A(x)=\frac{B_{0}}{2 q R^{2}}\left(q R_{0} x_{1} x_{3}-x_{2} r^{2}, \quad q R_{0} x_{2} x_{3}+x_{1} r^{2}, \quad-q R^{2} R_{0} \log \left(R / R_{0}\right)\right)^{\top},
$$




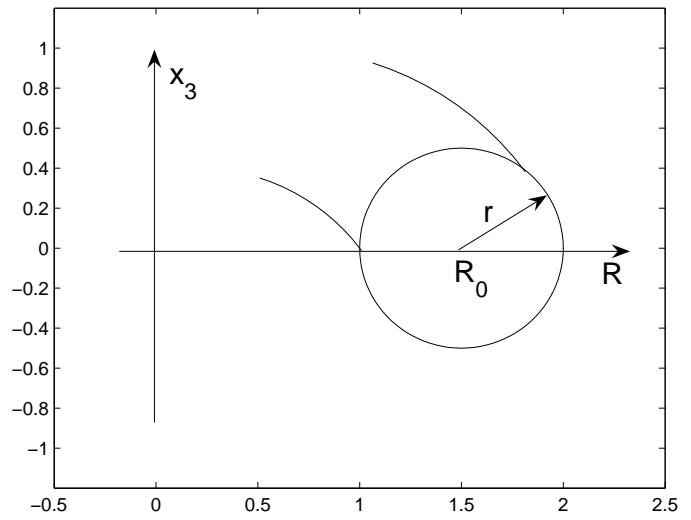

Figure 2: 2D tokamak geometry with circular concentric flux surfaces

where $B_{0}$ is the magnetic field on the axis and the constant $q$ is the safety factor, from which one obtains

$$
\begin{aligned}
B(x) & =\frac{B_{0}}{q R^{2}}\left(-x_{1} x_{3}-q R_{0} x_{2}, \quad-x_{2} x_{3}+q R_{0} x_{1}, \quad R\left(R-R_{0}\right)\right)^{\top}, \\
\|B(x)\| & =\frac{B_{0}}{q R} \sqrt{r^{2}+q^{2} R_{0}^{2}}, \\
b(x) & =\frac{1}{R \sqrt{r^{2}+q^{2} R_{0}^{2}}}\left(-x_{1} x_{3}-q R_{0} x_{2}, \quad-x_{2} x_{3}+q R_{0} x_{1}, \quad R\left(R-R_{0}\right)\right)^{\top},
\end{aligned}
$$

In the numerical tests, we shall choose the parameters as follows:

$$
R_{0}=1, \quad B_{0}=1, \quad q=2, \quad \mu=2.25 \cdot 10^{-6} .
$$

Moreover, we shall choose the initial condition as (see (4))

$$
y_{0}=\left(\begin{array}{llll}
1.05, & 0, \quad 0, \quad u_{0}
\end{array}\right)^{\top}
$$

by considering the following two values of $u_{0}$ :

- $u_{0}=0.0008117$, generating a transit orbit, i.e., a circular orbit, in the coordinates 55).

- $u_{0}=0.0004306$, which generates a banana shaped orbit, in the coordinates (55);

The 3D trajectories in the interval $\left[0,10^{6}\right]$ are respectively shown in the left plots of Figures 3,4 whereas those in the coordinates (55) are shown in the corresponding right plots.

We now further show that the higher-order LIMs are much more efficient than the lower-order ones. In fact, by selecting $k$ large enough so that energy-conservation is granted even for relatively large stepsizes, e.g. $k=20$, the methods can be regarded as spectral methods in time for large values of $s$ (but smaller than $k$ ). Spectral methods in time proved to be very effective in many instances, 
Table 3: Numerical results when solving the transit orbit $\left(h=8 \cdot 10^{3}\right)$ and the banana orbit $\left(h=10^{4}\right)$ tokamak problems on the interval $\left[0,10^{8}\right]$ by using the energy-conserving $\operatorname{LIM}(s, 20, s)$ methods (*** means non convergence of the nonlinear iteration, times are in sec).

\begin{tabular}{|r|rrr|rrr|}
\hline & \multicolumn{4}{|c|}{ transit orbit } & \multicolumn{3}{|c|}{ banana orbit } \\
\hline$s$ & iterations & time & error & iterations & time & error \\
\hline 1 & $* * *$ & $* * *$ & $* * *$ & $* * *$ & $* * *$ & $* * *$ \\
2 & $* * *$ & $* * *$ & $* * *$ & $* * *$ & $* * *$ & $* * *$ \\
3 & $* * *$ & $* * *$ & $* * *$ & $* * *$ & $* * *$ & $* * *$ \\
4 & $* * *$ & $* * *$ & $* * *$ & $* * *$ & $* * *$ & $* * *$ \\
5 & $* * *$ & $* * *$ & $* * *$ & $* * *$ & $* * *$ & $* * *$ \\
6 & $* * *$ & $* * *$ & $* * *$ & $* * *$ & $* * *$ & $* * *$ \\
7 & $* * *$ & $* * *$ & $* * *$ & $* * *$ & $* * *$ & $* * *$ \\
8 & $* * *$ & $* * *$ & $* * *$ & 773705 & 84.7 & $3.1 \mathrm{e} 00$ \\
9 & 1018824 & 114.4 & $3.0 \mathrm{e} 00$ & 570191 & 64.4 & $8.5 \mathrm{e}-01$ \\
10 & 734527 & 85.0 & $1.2 \mathrm{e} 00$ & 494422 & 57.2 & $6.2 \mathrm{e}-02$ \\
11 & 625527 & 74.2 & $1.1 \mathrm{e}-01$ & 457523 & 54.2 & $1.6 \mathrm{e}-02$ \\
12 & 569554 & 68.6 & $9.2 \mathrm{e}-03$ & 436163 & 52.6 & $1.3 \mathrm{e}-03$ \\
13 & 533843 & 65.7 & $7.1 \mathrm{e}-04$ & 419205 & 51.7 & $1.9 \mathrm{e}-04$ \\
14 & 509484 & 63.9 & $5.0 \mathrm{e}-05$ & 410197 & 51.7 & $1.1 \mathrm{e}-05$ \\
15 & 501218 & 64.1 & $2.5 \mathrm{e}-06$ & 402775 & 51.4 & $1.3 \mathrm{e}-06$ \\
16 & 493683 & 63.9 & $8.1 \mathrm{e}-07$ & 399053 & 51.6 & $2.5 \mathrm{e}-07$ \\
\hline
\end{tabular}

such as highly-oscillatory problems [20, stiff-oscillatory problems [10, fractional equations [1], and other problems $\left[21,8\right.$ For this purpose, let us solve the transit orbit problem on the interval $\left[0,10^{8}\right]$, by using a timestep as large as $h=8 \cdot 10^{3}$, and the banana orbit problem, on the same interval, by using a timestep $h=10^{4}$. In Table 3 we list the obtained results by using the $\operatorname{LIM}(s, 20, s)$ method, $s=1, \ldots, 16$. For both problems, a reference solution has been computed by using the $\operatorname{LIM}(18,20,18)$ method with the same timestep. In the table, one has, for each selected value of $s$ :

- *** if the nonlinear iteration (48) does not converge;

- the total number of nonlinear iterations 48 for covering the iteration interval;

- the corresponding execution time (in sec);

- the maximum error w.r.t. the reference solution.

As one may see, only for $s$ large enough the problems are solved. Moreover, even though each iteration (48) has a cost which increases with $s$, nevertheless, the higher $s$, the smaller the number of iterations needed, so that the overall execution time decreases with $s$, and so does the error. It is worth noticing that this effectiveness is made possible provided that a vector function is used for evaluating $S(y)$ and $\nabla H(y)$, as in the present case. In fact, this allows to exploit the vector architecture of nowadays processors $9^{9}$ The obtained results clearly testify the effectiveness of higher-order energy-conserving LIMs w.r.t. lower-order ones.

\footnotetext{
${ }^{8}$ The reasons for their effectiveness has been studied in 3 .

${ }^{9} \mathrm{~A}$ similar remark was observed in [20, Remark 3].
} 

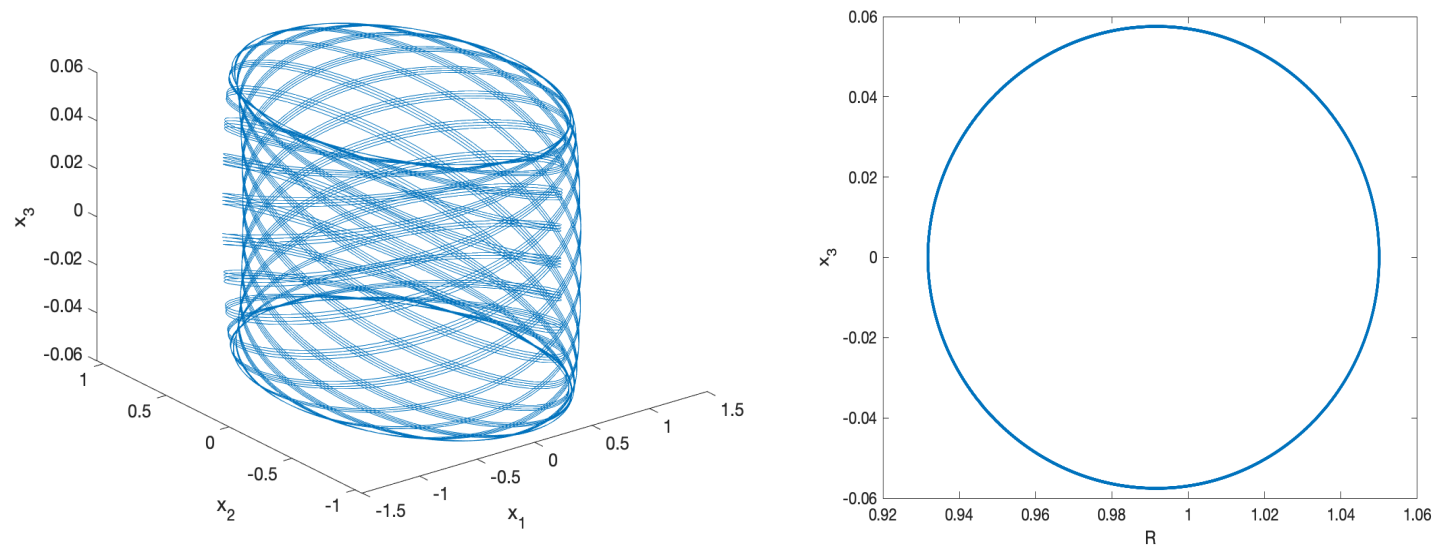

Figure 3: Transit orbit. Left plot: solution trajectory in the phase space; right plot: solution in the coordinates (55).
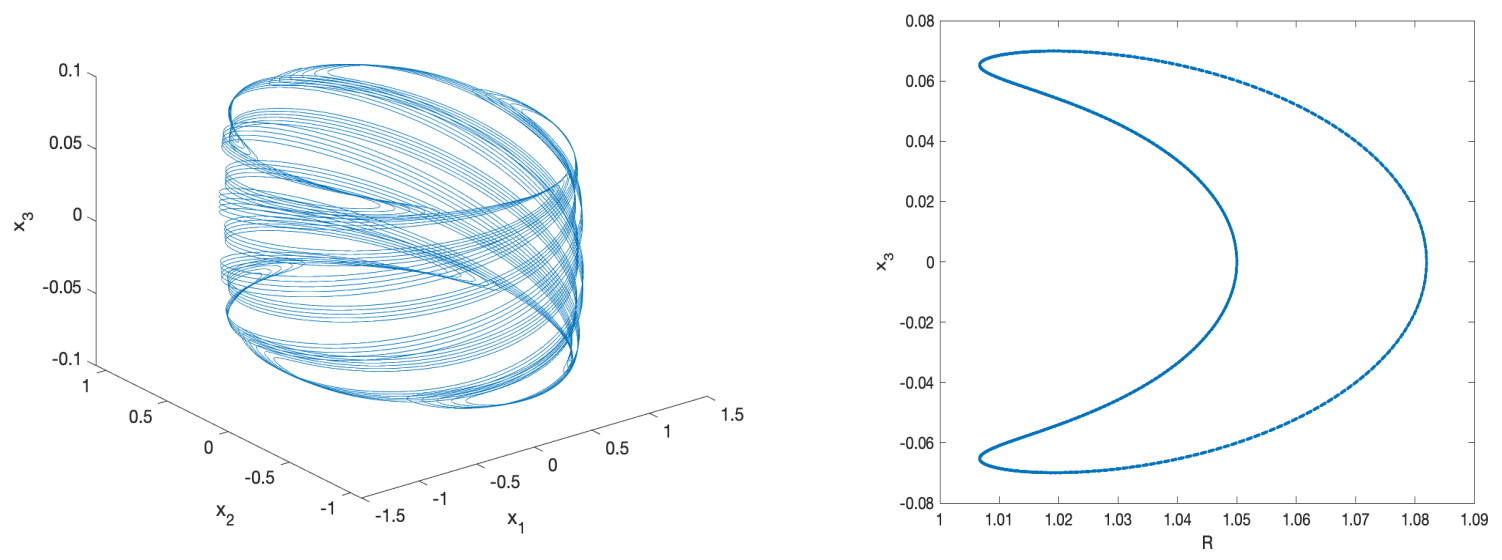

Figure 4: Banana orbit. Left plot: solution trajectory in the phase space; right plot: solution in the coordinates (55). 
Table 4: Dipole magnetic field with quadratic electric field problem solved on an interval containing $\left[0,10^{3}\right]$; (approximate) maximum timestep $\left(h_{\max }\right)$ allowed by the iteration (either fixed-point (f-p) or blended (blend)), total number of iterations (it), mean number of iterations per step (it/step), and execution times (in $s e c$ ).

\begin{tabular}{|r|rr|rr|rr|rr|rr|}
\hline & \multicolumn{2}{|c|}{ LIM(1,7,1) } & \multicolumn{2}{|c|}{$\operatorname{LIM}(2,8,2)$} & \multicolumn{2}{|c|}{$\operatorname{LIM}(3,9,3)$} & \multicolumn{2}{|c|}{$\operatorname{LIM}(4,9,4)$} & \multicolumn{2}{|c|}{ LIM $(5,9,5)$} \\
\hline iteration & $\mathrm{f}-\mathrm{p}$ & blend & $\mathrm{f}-\mathrm{p}$ & blend & $\mathrm{f}-\mathrm{p}$ & blend & $\mathrm{f}-\mathrm{p}$ & blend & \multicolumn{1}{|c|}{ f-p } & blend \\
\hline$h_{\text {max }}$ & .01 & 47 & .02 & 72 & .04 & 86 & .05 & 103 & .06 & 120 \\
it & 4329357 & 880 & 2697596 & 1120 & 5013330 & 1333 & 3225342 & 1420 & 2713788 & 1599 \\
it/step & 43.3 & 40 & 54.0 & 80 & 200.5 & 111.1 & 161.3 & 142.0 & 162.8 & 177.6 \\
time & 282.4 & 0.1 & 307.3 & 0.1 & 605.7 & 0.2 & 392.0 & 0.2 & 345.0 & 0.2 \\
\hline
\end{tabular}

Dipole magnetic field with quadratic electric field. We now consider a further problem, aimed at showing the potentialities of the blended iteration (51). This problem is still defined by (52)-54, with the initial condition replaced by

$$
y(0)=(1,1,0.01,0.01)^{\top} .
$$

Moreover, now the electric potential defining the Hamiltonian (6) is not zero, and is given by

$$
\phi(x)=\frac{1}{2} x^{\top} G x, \quad G=\operatorname{diag}\left(1,1,10^{4}\right) .
$$

According to the result of Theorem 6, now the fixed-point iteration may encounter stepsize restrictions. In Table 4 we list the obtained results by solving the problem on the smallest interval containing $\left[0,10^{3}\right]$, commensurable with the (approximately) maximum timestep $h_{\max }$ allowed by the used iteration, either fixed-point (f-p) or blended (blend), using the same methods considered in Table 2 In such a case, as is clear, we are not discussing accuracy but, instead, we want to emphasize the robustness of the nonlinear iteration 10

From the results listed in Table 4, one may observe that, for $\operatorname{LIM}(s, k, s)$ methods used for solving this problem:

- the maximum allowed timestep, $h_{\max }$, increases with $s$;

- the mean number of iterations per step increases with $h_{\max }$ (and, then, with $s$ );

- the blended iteration allows using much larger (indeed, huge) timesteps, w.r.t. the fixed-point iteration, resulting in much smaller execution times.

\subsection{Conclusions}

In this paper we have developed arbitrarily high-order methods for Poisson problems, with a major emphasis on the simulation of the gyrocenter dynamics of a charged particle in a constant magnetic field, which is a relevant problem in plasma physics. The methods are derived and studied within the framework of line integral methods, and their efficient implementation has been also sketched. The reported numerical tests duly confirms the theoretical achievements. As a future direction of

\footnotetext{
${ }^{10}$ Remarkably enough, all methods remain still energy-conserving, even when using large timesteps.
} 
investigations, we plan to study the speed-up of the convergence of the nonlinear iteration, when large value of $s$ are taken into account, corresponding to the use of the methods as spectral methods in time.

\section{Acknowledgements}

The research of the third author was supported by the National Key Research and Development Program (Grant No. 2017YFE0301700).

\section{References}

[1] P. Amodio, L. Brugnano, F. Iavernaro. A note on the continuous-stage Runge-Kutta(-Nyström) formulation of Hamiltonian Boundary Value Methods (HBVMs). Appl. Math. Comput. 363 (2019) 124634 https://doi.org/10.1016/j .amc.2019.124634

[2] P. Amodio, L. Brugnano, F. Iavernaro. Spectrally accurate solutions of nonlinear fractional initial value problems. AIP Conference Proceedings 2116 (2019) 140005 https://doi.org/10. $1063 / 1.5114132$

[3] P. Amodio, L. Brugnano, F. Iavernaro. Analysis of Spectral Hamiltonian Boundary Value Methods (SHBVMs) for the numerical solution of ODE problems. Numer. Algor. (2019) 1-20 https://doi.org/10.1007/s11075-019-00733-7

[4] L. Brugnano. Blended Block BVMs $\left(\mathrm{B}_{3} \mathrm{VMs}\right)$ : a family of economical implicit methods for ODEs. J. Comput. Appl. Math. 116 (2000) 41-62.

[5] L. Brugnano, M. Calvo, J.I. Montijano, L. Rández. Energy preserving methods for Poisson systems. J. Comput. Appl. Math. 236 (2012) 3890-3904.

[6] L. Brugnano, G. Frasca-Caccia, F. Iavernaro. Efficient implementation of Gauss collocation and Hamiltonian Boundary Value Methods. Numer. Algorithms 65 (2014) 633-650.

[7] L. Brugnano, G. Frasca-Caccia, F. Iavernaro. Line Integral Solution of Hamiltonian PDEs. Mathematics 7(3) (2019) article n. 275 https://doi.org/10.3390/math7030275

[8] L. Brugnano, F. Iavernaro. Line Integral Methods for Conservative Problems. Chapman and Hall/CRC, Boca Raton, FL, 2016.

[9] L. Brugnano, F. Iavernaro. Line Integral Solution of Differential Problems. Axioms 7(2) (2018) article n. 36. http://dx.doi.org//10.3390/axioms7020036

[10] L. Brugnano, F. Iavernaro, J.I. Montijano, L. Rández. Spectrally accurate space-time solution of Hamiltonian PDEs. Numer. Algorithms 81, no. 4 (2019) 1183-1202.

[11] L. Brugnano, F. Iavernaro, T. Susca. Hamiltonian BVMs (HBVMs): implementation details and applications. AIP Conf. Proc. 1168 (2009) 723-726.

[12] L. Brugnano, F. Iavernaro, D. Trigiante. Hamiltonian BVMs (HBVMs): A family of "driftfree" methods for integrating polynomial Hamiltonian systems. AIP Conf. Proc. 1168 (2009) $715-718$. 
[13] L. Brugnano, F. Iavernaro, D. Trigiante. Hamiltonian Boundary Value Methods (Energy Preserving Discrete Line Integral Methods). JNAIAM J. Numer. Anal. Ind. Appl. Math. 5, 1-2 (2010) 17-37.

[14] L. Brugnano, F. Iavernaro, D. Trigiante. A note on the efficient implementation of Hamiltonian BVMs. J. Comput. Appl. Math. 236 (2011) 375-383.

[15] L. Brugnano, F. Iavernaro, D. Trigiante. A simple framework for the derivation and analysis of effective one-step methods for ODEs. Appl. Math. Comput. 218 (2012) 8475-8485.

[16] L. Brugnano, C. Magherini. Blended Implementation of Block Implicit Methods for ODEs. Appl. Numer. Math. 42 (2002) 29-45.

[17] L. Brugnano, C. Magherini. The BiM Code for the Numerical Solution of ODEs. J. Comput. Appl. Math. 164-165 (2004) 145-158.

[18] L. Brugnano, C. Magherini, F. Mugnai. Blended Implicit Methods for the Numerical Solution of DAE Problems. J. Comput. Appl. Math. 189 (2006) 34-50.

[19] L. Brugnano, C. Magherini. Recent Advances in Linear Analysis of Convergence for Splittings for Solving ODE problems. Appl. Numer. Math. 59 (2009) 542-557.

[20] L. Brugnano, J.I. Montijano, L. Rández. On the effectiveness of spectral methods for the numerical solution of multi-frequency highly-oscillatory Hamiltonian problems. Numer. Algorithms 81, no. 1 (2019) 345-376.

[21] L. Brugnano, J.I. Montijano, L. Rández. High-order energy-conserving Line Integral Methods for charged particle dynamics. J. Comput. Phys. 396 (2019) 209-227.

[22] D. Cohen, E. Hairer. Linear energy-preserving integrators for Poisson systems. BIT Numer. Math. 51 (2011) 91-101.

[23] G. Dahlquist, Å. Björk. Numerical Methods in Scientific Computing. Volume I. SIAM, Philadelphia, PA, USA, 2008.

[24] F. Iavernaro, B. Pace. s-Stage Trapezoidal Methods for the Conservation of Hamiltonian Functions of Polynomial Type. AIP Conf. Proc. 936 (2007) 603-606.

[25] F. Iavernaro, B. Pace. Conservative Block-Boundary Value Methods for the Solution of Polynomial Hamiltonian Systems. AIP Conf. Proc. 1048 (2008) 888-891.

[26] F. Iavernaro, D. Trigiante. High-order symmetric schemes for the energy conservation of polynomial Hamiltonian problems. J. Numer. Anal. Ind. Appl. Math. 4, 1-2 (2009) 87-101.

[27] R. G. Littlejohn. Variational principles of guiding centre motion. Journal of Plasma Physics 29 (1983) 111-125.

[28] R. Zhang, J. Liu, H. Qin, Y. Tang. Energy-preserving algorithm for gyrocenter dynamics of charged particles. Numer. Algorithms 81 (2019) 1521-1530.

[29] R. Zhang, J. Liu, Y. Tang, H. Qin, J. Xiao, B. Zhu. Canonicalization and symplectic simulation of the gyrocenter dynamics in time- independent magnetic fields. Physics of Plasmas 21 (2014) 032504. http://dx.doi.org/10.1063/1.4867669 
[30] B. Zhu, Z. Hu, Y. Tang, R. Zhang. Symmetric and symplectic methods for gyrocenter dynamics in time-independent magnetic fields. Report No. ICMSEC-15-3, Institute of Computational Mathematics and Scientific/Engineering Computing Chinese Academy of Sciences, November 2015. 